(C) 2016 IEEE. Personal use of this material is permitted. Permission from IEEE must be obtained for all other uses, in any current or future media, including reprinting/republishing this material for advertising or promotional purposes, creating new collective works, for resale or redistribution to servers or lists, or reuse of any copyrighted component of this work in other works. 


\title{
Passive and Active Metamaterial-Inspired Nano-Scale Antennas
}

\author{
Richard W. Ziolkowski ${ }^{1,2}$ \\ ${ }^{1}$ Department of Electrical and Computer Engineering, University of Arizona, Tucson, AZ, USA, ziolkowski@ece.arizona.edu \\ ${ }^{2}$ School of Computing and Communications, University of Technology, Sydney (UTS), Sydney, Australia
}

\begin{abstract}
A variety of open and closed multi-layered nanoparticle structures have been considered analytically and numerically for their use as scatterers and radiators. These include metamaterial-inspired structures based on dielectrics and metals excited by either plane waves or electric Hertzian dipoles at optical frequencies. Both passive and active (gain impregnated dielectric) materials have been considered. Enhanced and mitigated scattering and radiating effects have been modeled. Nano-antenna and nano-amplifier configurations for optical applications have been emphasized. A review of these modeling efforts will be presented.
\end{abstract}

Index Terms-Gain, nano-antennas, nano-scale, optical antennas, radiators, scatterers

\section{INTRODUCTION}

There have been numerous metamaterial-inspired [1] and metamaterial-based [2] antenna designs considered at RF frequencies. Electrically small antenna designs have been realized with a single metamaterial unit cell and small sets of them; they have been shown to have many potential applications. The metamaterial-inspired, near field resonant parasitic (NFRP) paradigm also has been extended to visible wavelengths, leading to active ENZ optical metamaterials [3], as well as passive and active nano-antennas [4]-[8]; nanolasers [9], [10]; highly directive nano-antennas [10]-[12]; nano-amplifiers [13], [14]; and quantum jammers [13]-[15].

\section{NEAR-FIELD RESONANT PARASITIC RADIATORS}

One basic spherical geometry-based nanoantenna/amplifier configuration is shown in Fig. 1. A coreshell structure is driven/excited with an arbitrarily oriented and located electric Hertzian dipole (EHD) antenna. It, as well as the plane wave excitation case, is amenable to spherical mode expansion based solutions [4], [9], [13]; and thus, the problem can be treated with a Mie scattering problem solution approach. This type of canonical geometry is extremely useful because it allows for exploration of the basic radiation and scattering physics involved with these multi-layered structures. The corresponding infinite cylinder with plane wave and infinite line source excitations have also been considered with TE and TM modal expansions [5], [11].

The placement of the EHD source external to the coated nano-particle is an extension of the original metamaterialbased antenna design [16], which led to the metamaterial- inspired NFRP paradigm [17]. It results from the fact [4] that the EHD does not have to be internal to a resonant core-shell element to produce the enhanced total radiated power. As long as the dipole couples strongly with the resonator, the desired efficient, enhanced response occurs. By impregnating the dielectric core with gain, the resulting nano-antenna consists of the EHD excitation and a nano-amplifier core-shell. This system has a variety of nano-sensor applications, e.g., an exited molecule near a well-tuned nano-amplifier will have a very large far-field response.
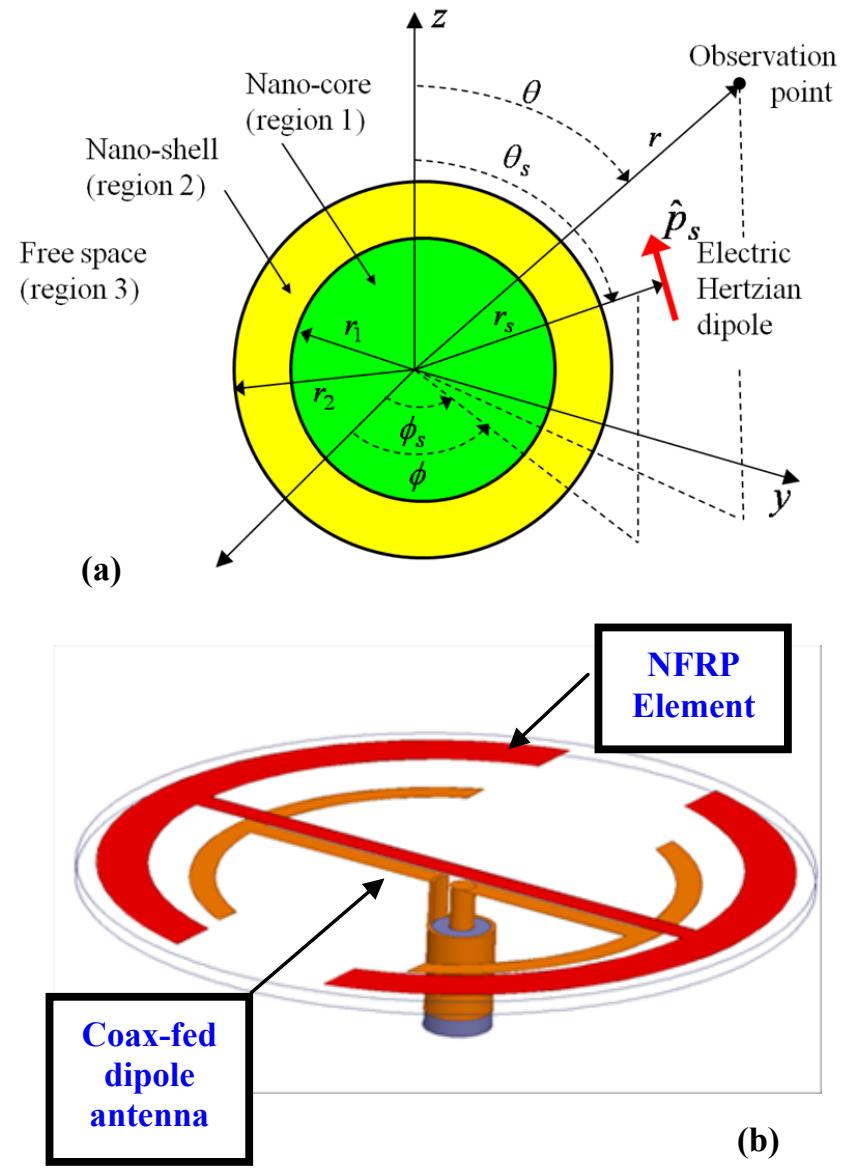

Fig. 1. Metamaterial-inspired NFRP paradigm. (a) An optical nano-antenna, i.e., a $30 \mathrm{~nm}$ radius core-shell (coated) nano-particle excited at $600 \mathrm{THz}$ by an EHD antenna [4], and (b) an electrically small, high efficiency, coax-fed 300 $\mathrm{MHz}$ Egyptian axe dipole antenna. 
This NFRP concept is further emphasized with the more familiar example of an Egyptian axe dipole (EAD) antenna, shown in Fig. 1(b). This electrically small antenna has a high radiation efficiency and is designed to be nearly completely matched to a $50 \Omega$ source at $300 \mathrm{MHz}$ as shown [18]. By augmenting the EAD antenna with a negative-impedance convertor (NIC)-based inductor, the resulting active NFRP antenna has a directivity-over-quality-factor ratio (D/Q) that surpasses the fundamental passive bounds [18], [19].

\section{MORE COMPLEX OPTICAL NANO-ANTENNAS}

Departures from canonical geometries require the introduction of models that can be simulated numerically. One such optical nano-antenna configuration studied in this manner is shown in Fig. 2. The passive and active versions of the coated open finite cylindrical structures shown in Fig. 2 were modeled with the frequency domain simulator in Microwave Studio CST [6]-[8]. This allowed for a numerical trick to incorporate active material in the core of the coated cylinder with this software package [6]. While an EHD excitation of the structure is shown in Fig. 2, the same model is trivially extended to a A plane wave excitation. Modeling both excitations using the same structural model allowed for immediate comparisons. Radiated power enhancements facilitated by the gain-impregnated core and enabled by the proper simultaneous tuning of the geometrical resonance and the dispersive gain resonance were found to be very similar to those obtained with the closed canonical structures.

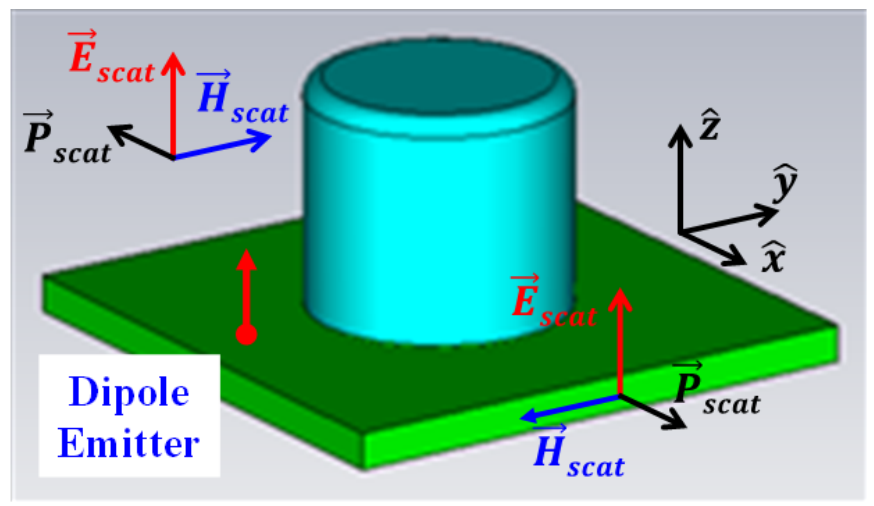

Fig. 2. CST model of a passive or active open cylinder excited by an EHD [6].

Other passive and active optical nano-antenna closed geometries, such as coated toroidal-shaped nano-particles have also been studied under plane wave and EHD excitations with these simulation environments [20]. The toroid structure is interesting because like the cylinder and unlike the sphere, it has a polarization dependent response. Similarly, the passive and active spherical coated nano-particle with a conical-based hole in its metallic coating that is shown in Fig. 3. This is another type of open optical nano-antenna structure. It was modeled in the frequency domain with the finite element solver in COMSOL Multiphysics [21]. This allowed the detailed study of both the near and far field radiated power distributions. The nuances of these numerical models and their results will be reviewed briefly in the presentation.

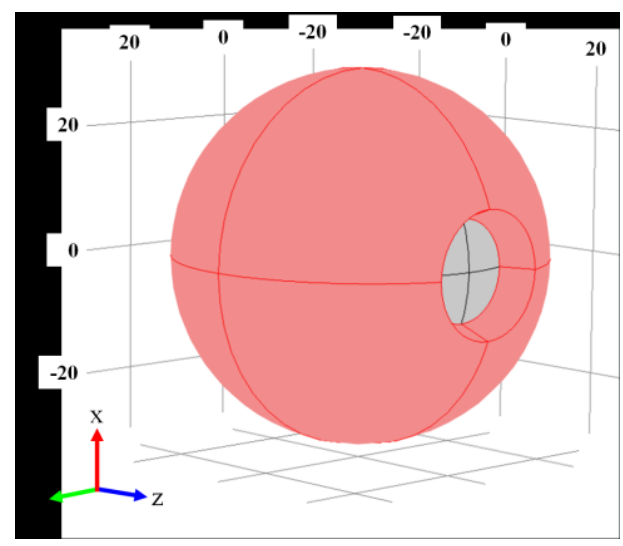

Fig. 3. COMSOL Multiphysics model of an open ( $40^{\circ}$ hole) spherical plasmonic shell with either a passive or active core. This optical nano-antenna channels its scattered power into a well-confined near-field beam.

\section{Microwave to Optical HuYGens Sources}

The juxtaposition of an electric dipole antenna and a magnetic dipole antenna leads the concept of a Huygens source. If the electric and magnetic dipole moments are properly weighted, the combined radiated fields will be directed into a preferred hemisphere with a large front-to-back ratio. An electrically small metamaterial-inspired microwave Huygens source is shown in Fig. 4a. It combines the Egyptian axe dipole (EAD) NFRP element with two protractor elements [1], i.e., two capacitively loaded loops. The EAD element produces the electric dipole field; the protractor elements generate the magnetic dipole field. The combination yields directive emission into the preferred hemisphere. The same concept can be achieved with nano-antennas. Using a gain impregnated multilayered core-shell particle, we have designed a Huygens source nanoparticle laser [22]. One set of layers produces an electric dipole mode; another set produces the magnetic dipole mode. The forward-directed field is amplified by the presence of the gain material in the core. This concept is illustrated in Fig. 4b.

\section{CONCLUDING REMARKS}

Many metamaterial concepts have led to interesting modifications of the properties of scatterers and radiators at microwave frequencies. They also have inspired immediate paths from their microwave proof-of-concept prototypes to their corresponding optical pathfinders. While the structures themselves can be quite different, the basic wave physics is very much the same. Moreover, while material properties are crucial for accurate designs in the microwave regime, they are even more critical for properly designing optical nanoantennas. Several metamaterial-inspired optical nano-antenna structures and their simulated behaviors will be reviewed in my presentation. 


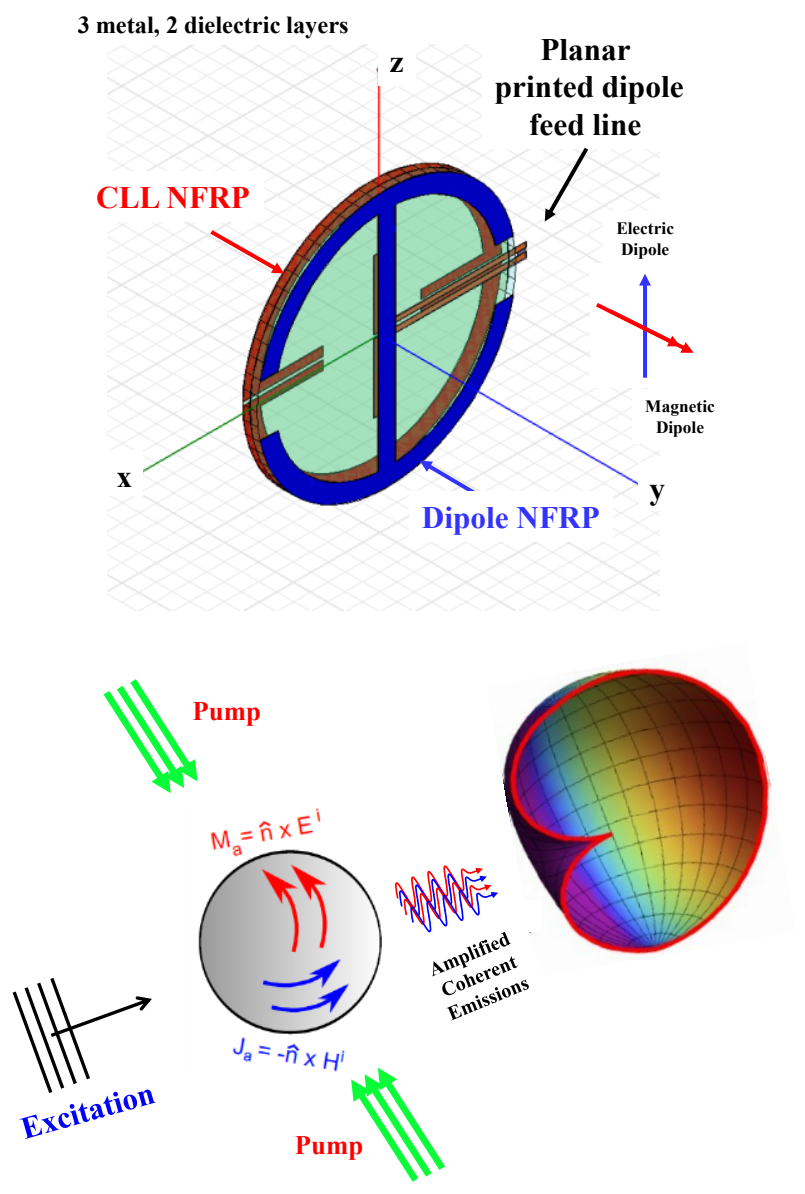

Fig. 4. Huygens sources. (a) Microwave combination of an electric (blue, along $\mathrm{z}$ axis) and a magnetic (red, along y axis) dipole NFRP antenna produces fields directed along the $\mathrm{x}$ direction; and (b) gain impregnated three layer core-shell nanoparticle combines electric and magnetic dipole modes to enable a Huygens source nanoparticle laser.

\section{REFERENCES}

[1] R. W. Ziolkowski, P. Jin and C.-C. Lin, "Metamaterial-inspired engineering of antennas," Proc. IEEE, vol. 99, pp. 1720-1731, Oct. 2011.

[2] Y. Dong and T. Itoh, "Metamaterial-based antennas," Proc. IEEE, vol. 100, pp. 2271-2285, Jul. 2012.

[3] J. A. Gordon and R. W. Ziolkowski, "CNP optical metamaterials," Opt. Express, vol. 16, no. 9, pp. 6692-6716, April 2008.

[4] S. Arslanagić, R. W. Ziolkowski, and O. Breinbjerg, "Radiation properties of an electric Hertzian dipole located near-by concentric metamaterial spheres" Radio Science, 42, RS6S16, doi:10.1029/2007RS003663, November 2007.
[5] S. Arslanagić, R. W. Ziolkowski, and O. Breinbjerg, "Analytical and numerical investigation of the radiation and scattering from concentric metamaterial cylinders excited by an electric line source," Radio Science, 42, RS6S15, doi:10.1029/2007RS003644, November 2007.

[6] J. Geng, R. W. Ziolkowski, R. Jin and X. Liang, "Numerical study of active open cylindrical coated nano-particle antennas," IEEE Photon., vol. 3, issue 6, 1093-1110, Dec. 2011.

[7] J. Geng, R. W. Ziolkowski, R. Jin and X. Liang, "Detailed performance characteristics of vertically polarized, cylindrical, active coated nanoparticle antennas," Rad. Sci., vol. 47, RS2013, doi:10.1029/2011RS004898, Apr. 2012.

[8] J. Geng, R. Jin, X. Liang and R. W. Ziolkowski, "Active cylindrical coated nano-particle antennas: Polarization-dependent scattering properties," J. Electromagnet. Wave Appl. (JEMWA), DOI:10.1080/09205071.2013.809669, June 2013.

[9] J. A. Gordon and R. W. Ziolkowski, "The design and simulated performance of a coated nano-particle laser," Opt. Express, vol. 15, Issue 5, pp. 2622-2653, March 2007.

[10] I. Liberal, I. Ederra, R. Gonzalo, and R. W. Ziolkowski, "Induction theorem analysis of resonant nanoparticles: Design of a Huygens source nanoparticle laser," Phys. Rev. Applied, vol. 1, 044002, May 2014.

[11] S. Arslanagić and R. W. Ziolkowski, "Directive properties of active coated nano-particles," Advanced Electromagnetics, vol. 1, no. 1, pp. 57-64, May 2012.

[12] S. D. Campbell and R. W. Ziolkowski, "Simultaneous excitation of electric and magnetic dipole modes in a resonant core-shell particle at infrared frequencies to achieve minimal backscattering," IEEE J. Sel. Topics Quantum Electron., vol. 19, no. 3, 4700209, May/June 2013.

[13] S. Arslanagić and R. W. Ziolkowski, "Active coated nano-particle excited by an arbitrarily located electric Hertzian dipole - resonance and transparency effects," J. Opt., vol. 12, 024014, Feb. 2010.

[14] S. Arslanagić and R. W. Ziolkowski, "Jamming of quantum emitters by active coated nano-particles," IEEE J. Selected Topics in Quantum Electronics, vol. 19, no. 3, 4800506, May/June 2013.

[15] S. Arslanagić and R. W. Ziolkowski, "Influence of active nano particle size and material composition on multiple quantum emitter enhancements: Their enhancement and jamming effects," Prog. Electromagn. Res., vol. 149, pp. 85-99, 2014.

[16] R. W. Ziolkowski and A. Erentok, "Metamaterial-based efficient electrically small antennas," IEEE Trans. Antennas Propagat., vol. 54, pp. 2113-2130, July 2006.

[17] A. Erentok and R. W. Ziolkowski, "Metamaterial-inspired efficient electrically-small antennas," IEEE Trans. Antennas Propag., vol. 56, no. 3, pp. 691-707, March 2008.

[18] N. Zhu and R. W. Ziolkowski, "Broad bandwidth, electrically small antenna augmented with an internal non-Foster element," IEEE Antennas Wireless Propag. Lett., vol. 11, pp. 1116-1120, 2012.

[19] N. Zhu and R. W. Ziolkowski, "Broad bandwidth, electrically small, non-Foster element-augmented antenna designs, analyses, and measurements," IEICE Transactions on Communications, vol. E96-B, no.10, pp. 2399-2409, Oct. 2013.

[20] J. Geng, D. Chen, R. Jin, X. Liang, J. Tang, and R.W. Ziolkowski, Study of active coated nano-toroid antennas, in Proc. 2013 International Workshop on Antenna Technology, iWAT2013, pp. 154-157, Karlsruhe, Germany, Mar. 2013.

[21] S. D. Campbell and R. W. Ziolkowski, "Near-field directive beams from passive and active asymmetric optical nano-antennas," IEEE J. Sel. Topics Quantum Electron., vol. 21, no. 4, 4800112, Jul./Aug. 2014.

[22] I. Liberal, I. Ederra, R. Gonzalo, and R. W. Ziolkowski, "Induction theorem analysis of resonant nanoparticles: Design of a Huygens source nanoparticle laser," Phys. Rev. Applied, vol. 1, 044002, May 2014. 